\title{
Adaptation and cross species interaction of Chromobacterium spp. within aquatic biofilms in temperate climate zone
}

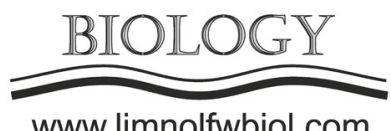

www.limnolfwbiol.com

\author{
Danilova K.V., Rikova V.S., Scherbakova A.A., Polyakov N.B., Solovyev A.I., \\ Egorova D.A.*
}

National Research Center of Epidemiology and Microbiology n. a. N.F. Gamaleya, Moscow, 123098, Russia

\begin{abstract}
Members of Chromobacterium genus are found in different natural aquatic reservoirs in tropical and subtropical climate zones. Chromobacterium species are considered as tropical bacterial species and are not resistant to cold temperatures during long-term storage in laboratory conditions. Here we present isolation of a resident strain of Chromobacterium vaccinii from root-associated biofilm of Carex spp. in Volkovskoye bog (Moscow region, Russia) and describe some unusual properties of isolated strain regarding adaptation to sub-zero temperatures and community lifestyle. While interaction and cooperation with other bacterial species within biofilm are important for resistance to sub-zero temperatures, we create MALDI-TOF MS based small molecule interaction network with other bacterial species.
\end{abstract}

Keywords: Chromobacterium, biofilm, social cheaters, cold-resistance, IDBac

\section{Introduction}

Members of Chromobacterium genus are considered as tropical/subtropical bacteria and only few reports describe environmental Chromobacterium species isolation in Europe: Chromobacterium violaceum in Poland (from Ixodes ricinus ticks) and an evidence of Chromobacterium vaccinii isolation from bog in Russia (Stojek and Dutkiewicz, 2004; Dobrovolskaya et al., 2020). Moreover, in cases of infections in Europe Chromobacterium species were discussed in prism of global warming, but primary sources of infection were not identified, and natural reservoirs of Chromobacterium species in Europe remain unexplored (Jędruszczak et al., 2019). Freezing water and ice coverage might provide significant stress to Chromobacterium species in natural reservoirs in case of climate zone with winter temperature below zero and it is still questionable if they can adopt and integrate into environmental microbial communities in temperate climate zone.

\section{Materials and Methods}

Biochemical properties of Chromobacterium vaccinii isolates were characterized using NEFERMtest 24 (Erbalachema) and ENDOtest (Erbalachema) and general microbiology techniques. MALDI-TOF MS were performed using an UltrafleXtreme mass-spectrometer (Bruker Daltonics) equipped with a smartbeam ${ }^{\mathrm{TM}}$-II laser (355 nm). NGS were performed on Illumina platform.
GC-MS analyses were carried out using Agilent 7820A (Agilent technologies) gas chromatograph with a Maestro MS detector (Interlab) with $30 \mathrm{~m} \times 0.25 \mathrm{~mm}$ i.d. capillary column Rxi-5ms (Restek).

\section{Results and discussion}

We have isolated unusual resident Chromobacterium vaccinii strains from root-associated biofilm of Carex spp. in Volkovskoye bog (Moscow region, Russia). Secure species identification of isolated Chromobacterium strains was based on whole genome sequencing. We described culturome of species isolated from the same biofilms using IDBac approach. Two phenotypically distinct Chromobacterium strains were identified - one of them represented irresponsible to inter- and intraspecies QS signals «social cheaters». Isolated strains did not survive exposure to subzero temperatures and changes of membrane fluidity was not enough as a trait to adopt to different temperatures, while in initial complex biofilm samples Chromobacterium vaccinii survived for month. We built a metabolic interaction network of small molecules produced by different species from the same with Chromobacterium biofilm and found close relationship with Serratia species. Altogether this data suggests an importance of deeper understanding of ecology and adaptability of Chromobacterium species to their natural habits and surrounding communities. 


\section{Acknowledgments}

This work was supported by grant MK-2241.2019.7 to DE.

\section{References}

Dobrovolskaya T.G., Golovchenko A.V., Yurchenko E.N. et al. 2020. Bacterial communities of regressive spots in ombrotrophic bogs: structure and functions. Microbiology 89:
107-114. DOI: 10.1134/S0026261720010063

Jędruszczak A., Węgrzyn-Bąk M., Budzyńska-Nosal R. et al. 2019. Sepsis caused by Chromobacterium violaceum - probably the first case in Europe, or Macbeth read anew. Annals of Agricultural and Environmental Medicine 26: 508-510. DOI: 10.26444/aaem/99295

Stojek N.M., Dutkiewicz J. 2004. Studies on the occurrence of Gram-negative bacteria in ticks: Ixodes ricinus as a potential vector of Pasteurella. Annals of Agricultural and Environmental Medicine 11: 319-322. 